\title{
Estudo de traumatismo craniencefálico experimental em ratos com aparelho de impacto cortical controlado
}

\author{
Amylcar Edemilson Dvilevicius ${ }^{1}$, Mirto Nelso Prandini², \\ Samuel Dobrowolski ${ }^{3}$, Ana Claudia Barbosa ${ }^{4}$ \\ Laboratório de Neuromicrocirurgia da Universidade Federal de São Paulo, \\ Escola Paulista de Medicina (Unifesp / EPM). São Paulo, SP, Brasil
}

\section{RESUMO}

Objetivo: Desenvolver um modelo de impacto cortical controlado (pneumático) para traumatismo craniencefálico experimental em ratos, sem exposição do encéfalo. Métodos: Com base em relatos da literatura, foi construído um modelo de impacto cortical pneumático, sendo testado em 30 ratos, com análise histopatológica de todos os animais. Resultados: Os resultados da análise histopatológica baseada na contagem de neurônios lesados por campo demonstraram a eficiência do modelo na produção de lesão traumática. Conclusão: Esse modelo para reprodução experimental de traumatismo craniencefálico poderá oferecer, por meio de metodologia reproduzivel e clinicamente relevante, situações patológicas causadas por forças mecânicas em lesões traumáticas cranianas.

\section{PALAVRAS-CHAVE}

Trauma craniencefálico. Modelo experimental em ratos.

\begin{abstract}
Experimental brain injury in rats with a controlled cortical impact device model

Objective: To develop a controlled cortical impact model (pneumatic) for experimental traumatic brain injury in rats, without brain exposition. Methods: Based in literature, we developed a pneumatic cortical impact model, and tested in thirty rats, with pathological analysis in all animals. Results: The results of pathological analysis of the studied animals, based in the quantification of the neurons injured for field, demonstrated the effectiveness of the model to produce experimental traumatic injury. Conclusion: This model for experimental traumatic brain injury study offers reproducible and clinically relevant pathological conditions through the application of mechanical forces in traumatic brain injury.
\end{abstract}

\section{KEY WORDS}

Traumatic brain injury. Experimental animal model.

\section{Introdução}

Este trabalho relata a construção e os testes iniciais de um modelo desenvolvido para reprodução de traumatismo craniencefálico de forma experimental.

Com o objetivo de produzir estudos experimentais envolvendo o traumatismo craniencefálico, houve dificuldade no acesso a um modelo para realização de estudos em animais. Optou-se, a partir daí, pela construção de um modelo que, dentro dos padrões de exigência científicos internacionais, pudesse realizar o traumatismo de forma controlável, reproduzível e fornecesse as lesões encefálicas compatíveis com as observadas nos traumatismos craniencefálicos fechados nos humanos. Realizou-se revisão ampla da literatura ${ }^{1-22}$ e convocou-se o apoio técnico necessário para auxiliar na execução de um projeto adequado.

1 Mestrando em Neurocirurgia pela Universidade Federal de São Paulo/Escola Paulista de Medicina (Unifesp/EPM), São Paulo, SP. Neurocirurgião do Hospital Angelina Caron, Campina Grande do Sul, PR, Santa Casa de Curitiba, Curitiba, PR, e Hospital Regional Vale do Ribeira, Pariquera-Açu, SP.

2 Chefe da cadeira de Neurocirurgia da Unifesp/EPM.

3 Neurocirurgião do Hospital Regional Vale do Ribeira, Pariquera-Açu, SP.

4 Patologista do Laboratório de Anatomia Patológica (LAP) - Registro, SP. 
Há grande quantidade de relatos sobre o desenvolvimento de modelos de traumatismo craniencefálico em animais, na tentativa de reproduzir aspectos das respostas biomecânicas, de síndromes neurológicas e outras condições patológicas observadas no traumatismo craniencefálico fechado. Entre os diversos modelos descritos, podem-se citar os modelos de aceleração ou rotação do crânio ${ }^{12}$, os de impacto induzido no crânio livre ${ }^{14}$ ou fixo ${ }^{9}$ ou aqueles com injeção rápida de solução salina através de pequena craniectomia, chamados de modelos de "percussão por fluido" 20 .

$\mathrm{O}$ aparato desenvolvido neste relato encaixa-se na categoria dos modelos de "impacto cortical controlado", em que, por meio de forças mecânicas (queda de peso ou pistão pneumático, por exemplo), provoca-se o trauma sobre a área de interesse no crânio do animal, podendo ser um crânio fechado ou com craniectomia prévia ${ }^{5}$. Optou-se pelo desenvolvimento de um impactor pneumático pela possibilidade de maiores variáveis nas análises biomecânicas, como o controle adequado do impacto (com possibilidade de variação na força do impacto e sua localização) e mudanças na rigidez da base sob a cabeça do rato (podendo-se criar variantes do traumatismo quanto à dissipação de energia). Também na literatura observou-se que as lesões obtidas com esse tipo de modelo de impacto cortical são mais parecidas com as encontradas em traumatismos fechados em humanos ${ }^{2,4,5,10,16,17}$.

\section{Material e métodos}

\section{Impactor}

Desenvolveu-se um pistão pneumático exclusivo para o modelo, baseado em descrições da literatura $2,5,7,10,14,16,17$, com $10,7 \mathrm{~cm}$ de altura e diâmetro de $19,5 \mathrm{~mm}$, podendo fazer um deslocamento de até $33 \mathrm{~mm}$. A extremidade do pistão (que atinge a cabeça do animal) tem regulagem, podendo variar de 6 a 10,5 mm. A movimentação do pistão (impactor) é feita a partir do compressor de ar ligado ao aparelho, podendo-se selecionar a pressão desejada pela observação do manômetro fixado no painel de controle, ao lado do botão de disparo, tendo como limite 200 psi (libras por polegada ao quadrado), com uma velocidade de impacto estimada em mais de $7 \mathrm{~m} / \mathrm{s}$. $O$ pistão fica mantido em base fixa, com regulagens de altura bilateral, diâmetro e rigidez da base (Figura 1).

\section{Preparação cirúrgica e lesão}

O trabalho foi aprovado pela Comissão de Ética da Universidade Federal de São Paulo (Unifesp), e os estudos, realizados no Laboratório de Neuromi-

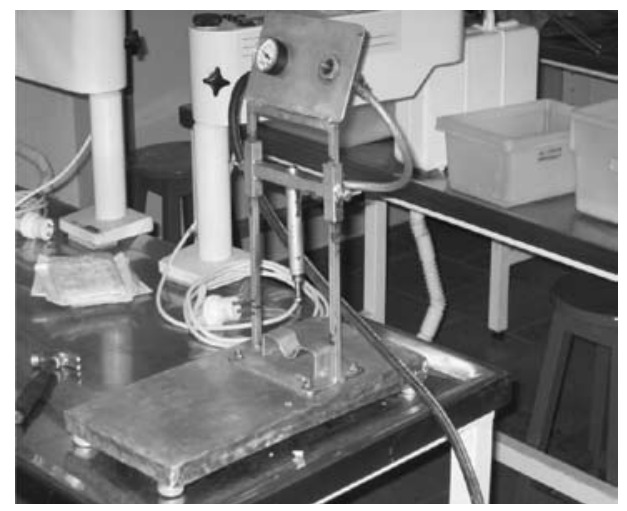

Figura 1-Modelo de dispositivo de impacto cortical controlado, conectado ao compressor de ar.

crocirurgia da Unifesp. Utilizaram-se 30 ratos da raça WISTAR/EPM, todos machos, com peso entre $250 \mathrm{e}$ $350 \mathrm{~g}$, mantidos em biotério da instituição, com boa ventilação e iluminação natural, com água e ração à vontade. Na preparação, foram colocados em gaiolas individuais, anestesiados com Zoletil $50^{\circledR}$ (cloridrato de tiletamina + cloridrato de zolazepam; Laboratório Virbac S/A, França) e posicionados adequadamente na base do aparelho. Foram utilizadas, sob a cabeça dos animais, bases de diferentes texturas: espuma, borracha e ferro (Tabela 1). Depois, fixou-se o crânio do animal com fita adesiva e realizou-se o traumatismo na região frontoparietal direita dos ratos (Figura 2). Foram submetidos a impacto que variou de 50 a 150 psi. Após o trauma, observaram-se as reações do rato, como as alterações respiratórias, cardíacas e crises convulsivas, sendo tomadas medidas de eventual reanimação. Passado um tempo de 60 a 90 minutos de observação em gaiola separada e aquecida a $30^{\circ} \mathrm{C}$, os animais foram novamente anestesiados e sacrificados por decapitação. Tiveram seus encéfalos removidos integralmente e fixados com solução de formol a $10 \%$ para posterior análise anatomopatológica.

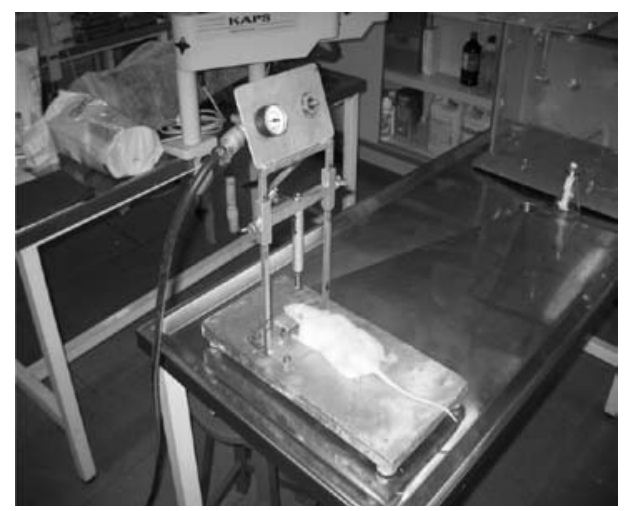

Figura 2 - Animal anestesiado no modelo de impacto cortical. 
Tabela 1

Relação dos animais, intensidade do impacto, base sob a cabeça, alterações clínicas imediatas e achados histopatológicos

\begin{tabular}{|c|c|c|c|c|}
\hline Animal $^{\mathrm{a}}$ & Impacto $^{\mathrm{b}}$ & Base $^{\mathrm{c}}$ & Eventos $^{d}$ & Patologia $^{\mathrm{e}}$ \\
\hline 1 & $50 \mathrm{psi}$ & Espuma & Não & 8 \\
\hline 2 & $50 \mathrm{psi}$ & Espuma & Não & 7 \\
\hline 3 & $60 \mathrm{psi}$ & Espuma & Não & 12 \\
\hline 4 & $60 \mathrm{psi}$ & Espuma & Não & 10 \\
\hline 5 & $70 \mathrm{psi}$ & Espuma & Não & 10 \\
\hline 6 & $70 \mathrm{psi}$ & Espuma & Não & 10 \\
\hline 7 & $70 \mathrm{psi}$ & Borracha & Não & 12 \\
\hline 8 & $80 \mathrm{psi}$ & Borracha & Não & 11 \\
\hline 9 & $90 \mathrm{psi}$ & Borracha & Não & 17 \\
\hline 10 & $90 \mathrm{psi}$ & Borracha & Não & 18 \\
\hline 11 & $100 \mathrm{psi}$ & Borracha & Não & 15 \\
\hline 12 & $100 \mathrm{psi}$ & Borracha & Não & 17 \\
\hline 13 & $100 \mathrm{psi}$ & Ferro & Bradicardia & 18 \\
\hline 14 & $110 \mathrm{psi}$ & Ferro & Não & 17 \\
\hline 15 & 110 psi & Ferro & Não & 18 \\
\hline 16 & $110 \mathrm{psi}$ & Ferro & Não & 23 \\
\hline 17 & 120 psi & Ferro & Bradicardia & 20 \\
\hline 18 & $120 \mathrm{psi}$ & Ferro & Não & 35 \\
\hline 19 & $120 \mathrm{psi}$ & Ferro & Não & 40 \\
\hline 20 & $130 \mathrm{psi}$ & Ferro & Dispnéia & 44 \\
\hline 21 & $130 \mathrm{psi}$ & Ferro & Dispnéia & 55 \\
\hline 22 & $130 \mathrm{psi}$ & Ferro & Crise convulsiva & 68 \\
\hline 23 & $140 \mathrm{psi}$ & Ferro & Crise convulsiva & 50 \\
\hline 24 & $140 \mathrm{psi}$ & Ferro & Dispnéia/apnéia & 85 \\
\hline 25 & $140 \mathrm{psi}$ & Ferro & Não & 72 \\
\hline 26 & $150 \mathrm{psi}$ & Ferro & Não & 86 \\
\hline 27 & $150 \mathrm{psi}$ & Ferro & Crise convulsiva & 90 \\
\hline 28 & $150 \mathrm{psi}$ & Ferro & Crise conv./apnéia & 85 \\
\hline 29 & $150 \mathrm{psi}$ & Ferro & Dispnéia & 83 \\
\hline 30 & $150 \mathrm{psi}$ & Ferro & Dispnéia/apnéia & 85 \\
\hline
\end{tabular}

\section{Análise anatomopatológica}

Após a fixação com formol a $10 \%$, os encéfalos foram cortados no plano coronal na espessura de $5 \mu \mathrm{e}$ corados com hematoxilina-eosina. Fizeram-se a análise geral das lesões e um estudo histológico, sob aumento de 400 vezes, que consistiu na contagem de neurônios lesados por campo de grande aumento. Em cada espécime foram feitas contagens em três campos de grande aumento; cada um desses campos continha, em média, 100 neurônios (Figura 3).

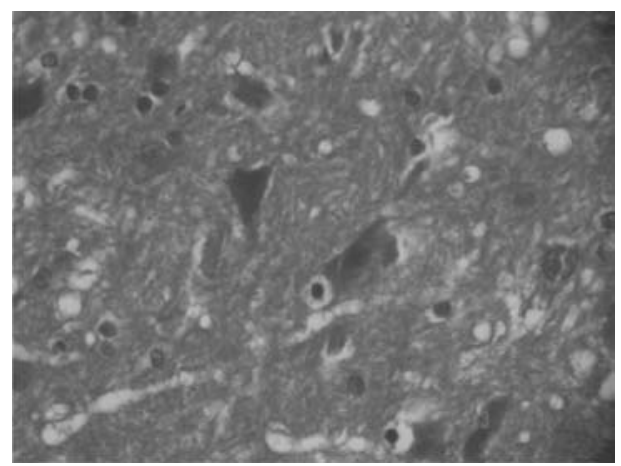

Figura 3-Análise histopatológica de animal submetido ao impacto cortical controlado, podendo-se observar neurônios lesados e edema cerebral.

\section{Resultados}

Observaram-se episódios de crises convulsivas focais em quatro ratos. Alterações respiratórias com dispnéia e/ou apnéia em cinco e bradicardia em dois ratos (Tabela 1). As alterações clínicas relatadas foram mais freqüentes nos animais submetidos a impacto de maior intensidade.

A principal alteração macroscópica observada durante a retirada do encéfalo dos espécimes foi a hemorragia subaracnóidea (HSA), vista principalmente na convexidade. Não se observaram fraturas de crânio, mesmo quando foi utilizado impacto mais potente.

Do ponto de vista microscópico, observaram-se alterações como: edema cerebral, petéquias hemorrágicas, contusão hemorrágica, congestão vascular, lesão axonal e neuronal. Com a finalidade de graduar a intensidade das lesões, foi feita a contagem de neurônios por campo de grande aumento, que demonstraram proporcionalidade da quantidade de neurônios lesados com o impacto utilizado no espécime (Tabela 1).

\section{Discussão}

$\mathrm{Na}$ literatura existem diversos relatos ${ }^{1,2,-7,10,12,16-18}$ sobre o desenvolvimento e a utilização de modelos para estudos experimentais no traumatismo craniencefálico. Entre os diversos tipos de aparatos, podem-se destacar dois grandes grupos: "percussão por fluido" e "impacto cortical controlado".

Os modelos de "percussão por fluido" utilizados em diversos relatos ${ }^{1,6,12,18}$ basicamente funcionam pela injeção de um pulso de líquido extradural através de uma cânula, causando uma deformação no cérebro por um período de 10 a 20 milissegundos ${ }^{12}$. Evidentemente alcançam o objetivo de reproduzir um número rele- 
vante de situações fisiopatológicas, porém têm duas importantes limitações: a restrição da investigação biomecânica (limitação na análise no pulso do líquido e suas possíveis dispersões no espaço extradural) e a de produzir, além da lesão axonal difusa e hemorragias intraparenquimatosas, lesões no tronco cerebral, condição esta que não é um achado primário freqüente nos traumatismos craniencefálicos graves em humanos. $\mathrm{E}$, com menor intensidade, produzir as contusões cerebrais, que são um achado mais comum entre as lesões cerebrais traumáticas 5 .

Já nos modelos de "impacto cortical controlado", podem-se dimensionar os eventos biomecânicos que contribuíram para a lesão, podendo estabelecer parâmetros como força, velocidade e deformação, além da magnitude da lesão tecidual e/ou funcional, dependendo do modelo. A maioria desses modelos usa pistões pneumáticos com extremidades adaptadas ao local de interesse do trauma, causando impactos precisos em sua velocidade e efeitos rápidos de deformação cortical controlada ${ }^{2,4,5,7,10,16,17}$. Existem também importantes diferenças entre os métodos para produção do impacto cortical controlado, podendo ser por "queda de peso" (não excedendo velocidade de $2 \mathrm{~m} / \mathrm{s}$ ) e o "pneumático", já mencionado e adotado neste estudo, que pode chegar a velocidades maiores que $7 \mathrm{~m} / \mathrm{s}$. Ambas as técnicas produzem lesões, porém a utilização do pistão pneumático, em virtude de sua maior velocidade e intensidade de impacto, propiciará lesões maiores e de maior gravidade ${ }^{4,5,7,13}$.

A opção por ratos fez-se em razão do maior número de modelos desenvolvidos para esses animais, o grande número de relatos quanto às suas respostas fisiológicas e comportamentais, compatibilidade com vários neurofármacos e anestésicos humanos, facilidade de acesso aos espécimes e seu custo.

Os resultados obtidos no trabalho são semelhantes aos de outros publicados na literatura, mostrando que a lesão obtida nos espécimes aumenta proporcionalmente à velocidade e à intensidade do impacto ${ }^{1-4,6-8,11,13,15,18,19,21,22}$. Os estudos foram iniciados, utilizando-se a intensidade de impacto de 50 psi (libras por polegada ao quadrado) e uma base de espuma macia sob a cabeça do animal. Gradualmente, foi elevada a intensidade do impacto até chegar a 150 psi e utilizando base de ferro sob a cabeça do animal, situação em que se encontrou o maior número de neurônios lesados por campo.

O impacto criado pelo modelo também provocou outros tipos de lesões habituais no traumatismo craniencefálico: contusões hemorrágicas, hemorragia subaracnóidea, petéquias hemorrágicas, lesões axonais e edema cerebral. Porém, a maioria dessas lesões é de difícil quantificação; a contagem de neurônios lesados observados em campos de grande aumento (400×), técnica descrita na literatura, é eficaz, acessível, de baixo custo e fácil quantificação. A partir do uso dessa técnica, verificou-se que, quanto maior a intensidade do impacto testado, maior a quantidade de neurônios lesados por campo ${ }^{13,14,21,22}$.

Até a intensidade de impacto de 150 psi, não foram observadas fraturas de crânio nos espécimes testados, apesar de não se utilizar capacete de proteção nos ratos, como em alguns relatos da literatura ${ }^{5,10}$. Para obter as lesões traumáticas também não foi necessária a realização de craniectomia prévia no local do impacto, condição também eventualmente utilizada.

As alterações sistêmicas decorrentes do traumatismo foram observadas e compatíveis com as relatadas em outros modelos, isto é, nos animais submetidos a maiores impactos; observaram-se, principalmente, bradicardia, dispnéia e apnéia. Isso aconteceu da mesma forma com as crises convulsivas focais verificadas nos animais submetidos também a impacto de maior intensidade.

O modelo construído oferece possibilidade de alteração da base sob a cabeça do animal, que dá margem a análises quanto à dissipação de energia do impacto ${ }^{16}$; podem-se também fazer outras alterações na extremidade do impactor, deixando-o maior que seus $10,5 \mathrm{~mm}$, aumentando, assim, a área de contato com o crânio, diminuindo-se o potencial de lesões localizadas e aumentando as lesões difusas ${ }^{10,13}$. Há, ainda, a possibilidade de eventuais mudanças na angulação da cabeça do espécime na base do modelo, podendo criar outras situações de interesse investigativo.

\section{Conclusão}

O modelo que desenvolvemos para estudar experimentalmente o impacto cortical controlado em ratos possibilita o estudo de muitos aspectos do traumatismo craniencefálico num simples aparato, fornecendo alto nível de controle biomecânico (alteração de base, seleção do local de impacto, possibilidade de eventuais angulações da cabeça do animal etc.), permitindo a mensuração morfológica e eventual evolução funcional.

Esse aparato mostrou-se efetivo na reprodução de achados patológicos observados nos traumatismos craniencefálicos fechados em humanos, como edema, lesão axonal e neuronal, fornecendo, de maneira proporcional, a intensidade do impacto, maior número de alterações, comprovadas pela contagem dos neurônios lesados por campo de grande aumento (400×).

Certamente será uma ferramenta efetiva para pesquisadores no estudo experimental dos traumatismos craniencefálicos. 


\section{Agradecimento}

Ao Sr. Dirlene Melo dos Santos pelas suas orientações técnicas, fundamentais para o desenvolvimento deste modelo.

\section{Referências}

1. CARBONELL WS, MARIS DO, MCCALL T, GRADY MS: Adaptation of the fluid percussion injury model to the mouse. J Neurotrauma 15:217-29, 1998.

2. CLARK RSB, KOCHANEK PM, DIXON CE et al.: Early neuropathologic effects of mild or moderate hypoxemia after controlled cortical impact injury in rats. J Neurotrauma 14:615-27, 1997.

3. CROCKARD HA, BROWN FD, CALICAAB et al.: Physiological consequences of experimental cerebral missile injury and use of data analysis to predict survival. J Neurosurg 46:784-94, 1977.

4. DENNY-BROWN D, RUSSEL WR: Experimental cerebral concussion. Brain 64:93-164, 1941.

5. DIXON CE, CLIFTON GL, LIGHTHALL JW et al: A controlled cortical impact modelo for traumatic brain injury in the rat. $J$ Neurosci Methods 39:253-62, 1991.

6. DIXON CE, LYETH BG, POVLISHOCK JT et al.: A fluid percussion model for experimental brain injury in the rat. $\mathrm{J}$ Neurosurg 67:110-9, 1987.

7. FOX GB, FAN L, LEVASSEUR RA, FADEN AI: Sustained sensory/motor and cognitive deficits with neuronal apoptosis following controlled cortical impact brain injury in the mouse. J Neurotrauma 15:599-614, 1998.

8. GURDJIAN ES, LISSNER HR, WEBSTER JE et al.: Studies on experimental concussion: relation of physiologic effect to time duration of intracranial pressure increase at impact. Neurology 4:674-81, 1954.

9. GURDJIAN ES, WEBSTER JE: Experimental head injury with special reference in the mechanical factors in acute trauma. Surg Gynecol Obstet 76:623-34, 1943.

10. MARMAROU A, MONTASSER A, FODA A et al.: A new model of diffuse brain injury in rats. Part I: Pathophysiology and biomechanics. J Neurosurg 80:291-300, 1994.

11. MATTIASSON GJ, PHILIPS MF, TOMASEVIC G et al.: The rotating pole test: avaluation of this effectiveness in assessing functional motor deficits following experimental head injury in the rat. J Neurosci Methods 95:75-82, 2000.
12. McINTOSH TK, VINK R, NOBLE L et al.: Traumatic brain injury in the rat: characterization of a lateral fluid-percussion model. Neuroscience 28:233-44, 1989.

13. MONTASSER A, FODA A, MARMAROU A: A new model for diffuse brain injury in rats. Part II: Morphological characterization. J Neurosurg 80:301-13, 1994.

14. NILSSON B, PONTEN U, VOIGT G: Experimental head injury in the rat. Part I: Mechanics, pathophysiology and morphology in an impact acceleration trauma model. J Neurosug 47:241-51, 1977.

15. PIERCE JES, SMITH DH, TROJANOWSKI JQ, McINTOSH TK: Enduring cognitive, neurobehavioral and histopathological changes persist for up to one year following severe experimental brain injury in rats. Neuroscience 87:359-69, 1998.

16. PIPER IR, THOMSON D, MILLER JD: Monitoring weight drop velocity and foam stiffness as an aid to quality control of a rodent model of impact acceleration neurotrauma. $J$ Neurosci Methods 69:171-4, 1996.

17. SCHEFF SW, BALDWIN AS, BROWN RW, KRAEMER PJ: Morris water maze deficits in rats following traumatic brain injury: lateral controlled cortical impact. J Neurotrauma 14:615-27, 1997

18. SHIMAK, MARMAROU A: Evaluation of brain stem dysfunction following severe fluid-percussion head injury to the cat. J Neurosurg 74:270-7, 1991.

19. SMITH DH, CHEN XH, PIERCE JE et al.: Progressive atrophy and neuron death for one year following brain trauma in the rat. J Neurotrauma 14:715-27, 1997.

20. SULLIVAN HG, MARTINEZ J, BECKER DP et al.: Fluidpercussion model of mechanical brain injury in the cat. $J$ Neurosurg 45:520-34, 1976.

21. TANG $Y$, NODA $Y$, HASEGAWA T, NABESHIMA T: A concussive-like brain injury model in mice (I): impairment in learning and memory. J Neurotrauma 14:851-62, 1997.

22. TANG $Y$, NODA $Y$, HASEGAWA T, NABESHIMA T: A concussive-like brain injury model in mice (II): selective neuronal loss in the cortex and hippocampus. J Neurotrauma 14:863-73, 1997.

Original recebido em dezembro de 2007

Aceito para publicação em abril de 2008

\section{Endereço para correspondência}

Amylcar Edemilson Dvilevicius

Avenida República do Libano, 370

82520-500 - Curitiba, PR

E-mail:amylcar@uol.com.br 